\title{
Peuplement de la faune de crevettes de la Forêt des Marais Tanoé-Ehy (Sud-Est de la Côte d'Ivoire)
}

\author{
Claver Kémomadjèhi Djiriéoulou*(1), Mamadou Bamba(1), Mexmin Koffi Konan(2), Gervais \\ Konan N'Zi(1), Gouli Gooré Bi(1), Tidiani Koné(3). \\ (1) Laboratoire d'Hydrobiologie, UFR Biosciences, Université Félix HOUPHOUËT-BOIGNY, 22 BP 582 \\ Abidjan 22, Côte d'Ivoire. \\ (2) Laboratoire d'Environnement et de Biologie Aquatique, UFR Sciences et Gestion de l'Environnement, \\ Université Nangui Abrogoua, 02 BP 801 Abidjan 02, Côte d'Ivoire. \\ (3) UFR Environnement, Université Jean LOROUGNON GUEDE, BP 150 Daloa, Côte d'Ivoire. \\ *E-mail de l'auteur correspondant : claverdjirieoulouk@yahoo.fr
}

Original submitted in on $6^{\text {th }}$ January 2017. Published online at www.m.elewa.org on 30 $0^{\text {th }}$ April 2017 https://dx.doi.org/10.4314/jab.v112i1.15

\section{RESUME}

Objectif : Cette étude se propose d'étudier l'écologie et la diversité des communautés de crevettes dans la Forêt des Marais Tanoé-Ehy (Sud-Est de la Côte d'Ivoire, Afrique de l'Ouest).

Méthode et résultats : Les échantillonnages ont été menés mensuellement, d'avril 2012 à mars 2013 à l'aide d'une épuisette et des nasses. Six espèces de crevettes ont été capturées: Desmocaris trispinosa (Desmocarididae), Macrobrachium dux, M. equidens, M. macrobrachion, M. vollenhovenii et M. thysi (Palaemonidae). Desmocaris trispinosa est la plus largement distribuée (100 \% d'occurrence et $92,27 \%$ d'abondance relative). L'analyse de redondance (RDA) a montré que les variables environnementales telles que le taux d'oxygène dissous, la conductivité, la température de l'eau, la transparence, les débris végétaux, les plantes aquatiques et le taux de fermeture de la canopée influencent fortement la diversité taxonomique.

Conclusion et application des résultats : Cette étude a permis de déterminer l'influence des variables environnementales sur la diversité et l'abondance de la faune de crevettes dans la Forêt des Marais Tanoé-Ehy et confirme la possibilité d'utiliser l'espèce $M$. thysi pour évaluer la qualité des habitats. Aussi, a-t-elle démontré le caractère exceptionnel de cette forêt par sa spécificité et recommande vivement sa préservation.

Mots clés : crevettes, écologie, diversité, Forêt des Marais de la Tanoé-Ehy, Côte d'Ivoire.

\section{Shrimps assemblage in the Tanoe-Ehy Swamp Forest South East Côte d'Ivoire) ABSTRACT}

Objective: This study aims to determine ecology and diversity of shrimps communities in the TanoeEhy Swamp Forest (South East Côte d'Ivoire, West Africa).

Method and results: Sampling was conducted monthly from April 2012 to March 2013 using a handled net and lobster pots. Overall, six species were captured: Desmocaris trispinosa (Desmocarididae), Macrobrachium dux, M. equidens, M. macrobrachion, M. vollenhovenii and M. Thysi (Palaemonidae). Desmocaris trispinosa was largest distributed with $100 \%$ of occurrence and $92.27 \%$ of abundance. The Redundancy Analysis (RDA) performed showed that environmental variables such as dissolved 
oxygen, conductivity, water temperature, water velocity, vegetal debris occurrence, aquatic plant and canopy closure influence strongly diversity and abundance of shrimp fauna.

Conclusion and applications of results: This study determined the influence of environmental variables on the diversity and abundance of shrimp fauna in the Tanoé-Ehy Marsh Forest and confirms the possibility of using species $M$. thysi to assess the quality of habitats. Therefore, it has demonstrated the exceptional character of this forest by its specificity and strongly recommends its preservation. Key-words: shrimps, ecology, diversity, Tanoe-Ehy Swamp Forest, Côte d'Ivoire.

\section{INTRODUCTION}

Parmi les macro-invertébrés peuplant les écosystèmes aquatiques, les crevettes constituent un groupe zoologique très diversifié (De Grave \& Fransen, 2011) dont 300 espèces présentent un intérêt économique (Fischer et al., 1981). En outre, elles jouent un rôle considérable dans le processus écologique des écosystèmes aquatiques, agissant à différents niveaux tropiques comme herbivores, détritivores, prédateurs et proies (Cartes et al., 2010). Par ailleurs, le cycle biologique de plusieurs espèces est caractérisé par des migrations entre milieux de salinités différentes (Rafalimanana, 2003). Le Sud-Est de la Côte d'Ivoire est une zone fortement anthropisée où les exploitations agricoles ont remplacé la végétation naturelle sur plus de la moitié de sa surface (Hauhouot, 2004). En effet, on y assiste à l'installation incontrôlée du phénomène de monoculture à cause de l'extension des palmeraies. Ce qui pourrait influencer négativement la biodiversité. Toutefois, il existe dans cette zone, une portion de forêt relativement intacte, la Forêt des Marais TanoéEhy (FMTE), qui est la plus grande forêt marécageuse de cette région. De par la présence de certaines espèces menacées, cette forêt a été identifiée comme un site à haute valeur pour la conservation (Koné et al., 2008). Nonobstant, elle subit diverses pressions anthropiques dont le braconnage, l'exploitation forestière abusive et les défrichements agricoles familiaux ou industriels (Konan, 2014). Toutes ces menaces qui pèsent sur cet hydrosystème

\section{MATERIEL ET METHODES}

Milieu d'étude : La Forêt des Marais Tanoé-Ehy (FMTE) est localisée dans la Région du Sud-Comoé, précisément dans le Département de Tiapoum entre les latitudes $5^{\circ} 06^{\prime}$ et $5^{\circ} 12^{\prime} \mathrm{N}$ et les longitudes $2^{\circ} 43^{\prime}$ ont suscité des investigations pluridisciplinaires, notamment en Primatologie (Béné \& Akpatou, 2007; Gonédélé Bi et al., 2008; Koné et al., 2008), en Ichtyologie (Koné et al., 2009; Konan, 2014), en Ornithologie (Ahon, 2010), en Botanique (Adou Yao, 2007; Missa et al., 2015) en Batrachologie (Kpan, 2012) afin de démontrer le caractère exceptionnel de cette forêt par sa spécificité, sa richesse et sa diversité faunique (zone de frayère pour de nombreuses espèces) et floristique en vue de sa sauvegarde. Par ailleurs, cette forêt est adjacente à un vaste plan d'eau lagunaire (complexe lagunaire Aby-Tendo-Ehy) qui lui sert d'intermédiaire avec l'océan atlantique et constitue le siège de la plus importante activité de pêcherie de la région. Durant les saisons pluvieuses, la FMTE est régulièrement inondée. Sa surface de contact avec le milieu lagunaire devient alors plus importante tandis qu'au cours des périodes d'étiage, une partie de ses eaux se retire dans le milieu lagunaire, laissant entrevoir d'importants échanges entre ces deux habitats (Konan, 2014). Du fait de cette contigüité, les eaux de la FMTE pourraient intervenir dans le cycle biologique de certaines espèces crevettes pour la phase de reproduction qui nécessite les eaux douces. Or, aucune donnée relative aux crevettes n'y existe. En conséquence, cette étude se propose-t-elle d'étudier la distribution spatio-temporelle de la faune de crevettes dans cet écosystème en vue d'une contribution à sa sauvegarde.

et $2^{\circ} 92^{\prime} \mathrm{W}$ (Figure 1). Elle s'étend aux Souspréfectures de Tiapoum, Noé et Nouamou en Côte d'ivoire. Couvrant une superficie de 12000 ha, elle est bordée, en partie, par la lagune Ehy à l'ouest et 
le fleuve Tanoé au sud et à l'est. Cette forêt marécageuse est entourée de plusieurs localités dont Nouamou, Dohouan, Kotoagnuan, Saykro, Atchimanou, Ehania-Tanoé, Kadjakro, KongodjanTanoé et Yao Akakro. C'est l'un des derniers refuges au monde pour au moins trois espèces de singes qui figurent sur la liste rouge de l'UICN comme espèces en danger critique d'extinction. II s'agit de Cercopithecus diana roloway (Linné, 1758), Cercocebus atys lunulatus (Audebert, 1797) et Piliocolobus badius waldronae (Kerr, 1792) (Koné et al., 2008).

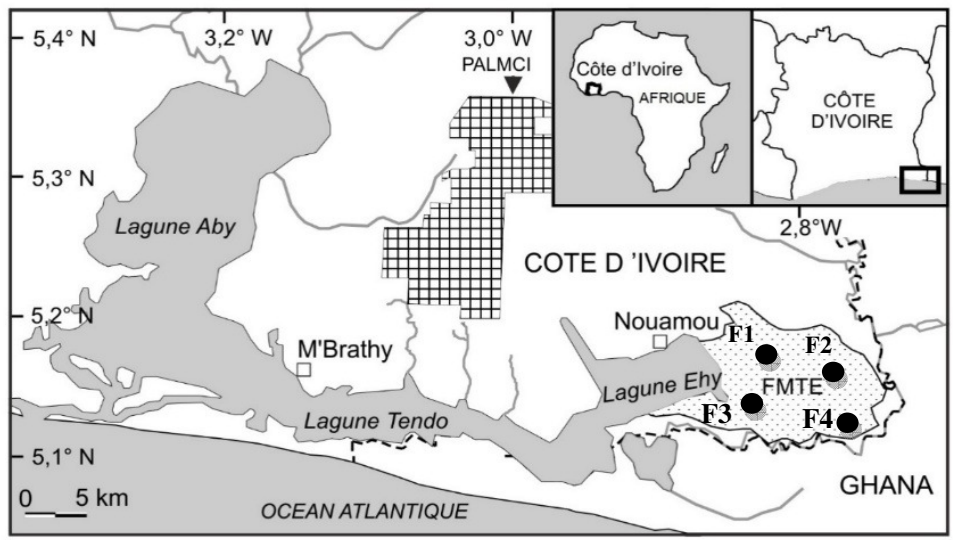

Figure 1 : Stations échantillonnées dans la Forêt des Marais Tanoé-Ehy (Côte d'Ivoire) d'avril 2012 à mars 2013.

Mesure des variables environnementales: Les paramètres physico-chimiques (Tableau 1) ont été mesurés mensuellement in situ d'avril 2012 à mars 2013 dans quatre stations. La température de l'eau, le $\mathrm{pH}$, la conductivité, le taux d'oxygène dissous ont été mesurés à l'aide d'un multiparamètre de marque Sper Scientifique. Outre ces appareils, un disque de Secchi (de $30 \mathrm{~cm}$ de diamètre peint en blanc-noir) et une barre graduée (en centimètres) ont été utilisés pour estimer, respectivement, la transparence et la profondeur de l'eau. Le taux moyen de fermeture de la canopée ainsi que la nature du substrat (mélange sable-gravier, boue, débris végétaux) ont été visuellement estimés en pourcentage pour chaque site conformément à la méthode de Gordon et al. (1994) et Rios \& Bailey (2006).

Échantillonnage et identification des crevettes : Les crevettes ont été échantillonnées mensuellement d'avril 2012 à mars 2013 à l'aide d'une épuisette (25 $\mathrm{cm}$ de diamètre d'ouverture et $2 \mathrm{~mm}$ de vide de maille). La pèche est effectuée par une seule personne munie d'un bracelet à chronomètre pendant $5 \mathrm{~min}$. Le chronomètre est mis en marche dès la plongée de l'épuisette dans l'eau et est arrêté à chaque retrait de cette dernière de l'eau pour le tri des spécimens capturés. Après le tri, le chronomètre est à nouveau mis en marche et la pêche se poursuit jusqu'à épuiser le timing. En plus de l'épuisette, des nasses appâtées par les résidus d'huiles de coco et les restes des aliments (poissons, viandes) ont été utilisées. Les crevettes capturées ont été conservées dans des bocaux contenant du formaldéhyde titré à $10 \%$ et étiquetés. L'étiquette porte la date et l'heure de la récolte, le nom de la station d'échantillonnage et le type d'engin de capture. L'identification des crevettes s'est faite à l'aide des clés de détermination de Monod (1966 et 1980), Powell (1982), Gooré Bi et al. (2002) et Short (2004). 
Tableau 1 : Variables environnementales (moyenne \pm écart type) mesurées dans les différentes stations (F1 : Ayénou 1 ; F2 : Ayénou 2 ; F3 : Dohouan ; F4 : Macrobo) dans la Forêt des Marais Tanoé-Ehy (Côte d-Ivoire) d'avril 2012 à mars 2013.

\begin{tabular}{|c|c|c|c|c|c|c|c|c|c|c|c|c|}
\hline \multirow{2}{*}{ Stations } & \multirow{2}{*}{ Saisons } & \multicolumn{11}{|c|}{ Variables environnementales } \\
\hline & & $\mathrm{pH}$ & $\mathrm{O}_{2}(\%)$ & Cnd $(\mu \mathrm{S} / \mathrm{cm})$ & Prof $(m)$ & $\mathrm{Te}\left({ }^{\circ} \mathrm{C}\right)$ & Trans (cm) & MSG & $B$ & DV & PA & FC \\
\hline \multirow{2}{*}{$\mathrm{F} 1$} & SP & $5,92 \pm 0,35$ & $22,07 \pm 6,01$ & $29,71 \pm 4,31$ & $1,47 \pm 0,54$ & $27,60 \pm 2,79$ & $19,86 \pm 4,10$ & \multirow{2}{*}{5} & \multirow{2}{*}{70} & \multirow{2}{*}{20} & \multirow{2}{*}{5} & \multirow{2}{*}{95} \\
\hline & SS & $6,22 \pm 1,19$ & $16,10 \pm 7,99$ & $23,83 \pm 5,15$ & $0,93 \pm 0,41$ & $29,48 \pm 3,38$ & $29,20 \pm 6,68$ & & & & & \\
\hline \multirow{2}{*}{ F2 } & $\mathrm{SP}$ & $6,63 \pm 0,52$ & $23,30 \pm 12,67$ & $44,69 \pm 11,65$ & $1,23 \pm 0,55$ & $26,11 \pm 1,95$ & $26,86 \pm 9,46$ & \multirow{2}{*}{5} & \multirow{2}{*}{75} & \multirow{2}{*}{15} & \multirow{2}{*}{5} & \multirow{2}{*}{90} \\
\hline & SS & $6,24 \pm 0,73$ & $21,64 \pm 7,63$ & $50,20 \pm 5,91$ & $0,65 \pm 0,23$ & $26,78 \pm 1,52$ & $35,00 \pm 6,82$ & & & & & \\
\hline \multirow{2}{*}{ F3 } & $\mathrm{SP}$ & $6,37 \pm 0,57$ & $38,91 \pm 6,29$ & $34,00 \pm 4,06$ & $2,25 \pm 0,58$ & $25,54 \pm 0,81$ & $50,57 \pm 8,94$ & \multirow{2}{*}{20} & \multirow{2}{*}{35} & \multirow{2}{*}{30} & \multirow{2}{*}{15} & \multirow{2}{*}{15} \\
\hline & SS & $6,22 \pm 1,05$ & $34,28 \pm 7,15$ & $36,12 \pm 4,95$ & $1,87 \pm 0,39$ & $25,90 \pm 0,75$ & $47,40 \pm 6,50$ & & & & & \\
\hline \multirow{2}{*}{ F4 } & $\mathrm{SP}$ & $6,11 \pm 0,70$ & $31,59 \pm 11,35$ & $39,74 \pm 4,56$ & $1,43 \pm 0,46$ & $25,97 \pm 1,18$ & $35,29 \pm 9,38$ & \multirow{2}{*}{10} & \multirow{2}{*}{30} & \multirow{2}{*}{50} & \multirow{2}{*}{10} & \multirow{2}{*}{95} \\
\hline & SS & $5,49 \pm 0,87$ & $24,52 \pm 5,54$ & $37,20 \pm 8,38$ & $1,02 \pm 0,22$ & $26,46 \pm 1,29$ & $35,60 \pm 58$ & & & & & \\
\hline
\end{tabular}


Analyse des données : La richesse taxonomique et le pourcentage d'occurrence ont été utilisés pour déterminer la structure et la dynamique du peuplement de crevettes. Le pourcentage d'occurrence est définie par la formule suivante : $\% \mathrm{OF}=(\mathrm{pi} / \mathrm{P}) \times 100$, avec $\mathrm{pi}:$ nombre d'échantillons où l'espèce $\mathrm{i}$ apparaît et $\mathrm{P}$ : nombre total d'échantillons dans un milieu. La classification des taxons sur la base de leur pourcentage d'occurrence a été faite selon Dajoz (2000) : \%OF $\geq 50$ : taxon constant ; $25 \leq \% \mathrm{OF}<50$ : taxon accessoire et $\% \mathrm{OF}$ $<25$ : taxon accidentel. Les variations spatiotemporelles de la richesse spécifique ont été

\section{RESULTATS}

Composition et distribution des espèces

Pourcentage d'occurrence: Six espèces de crevettes ont été échantillonnées dans l'ensemble des quatre stations prospectées: Desmocaris
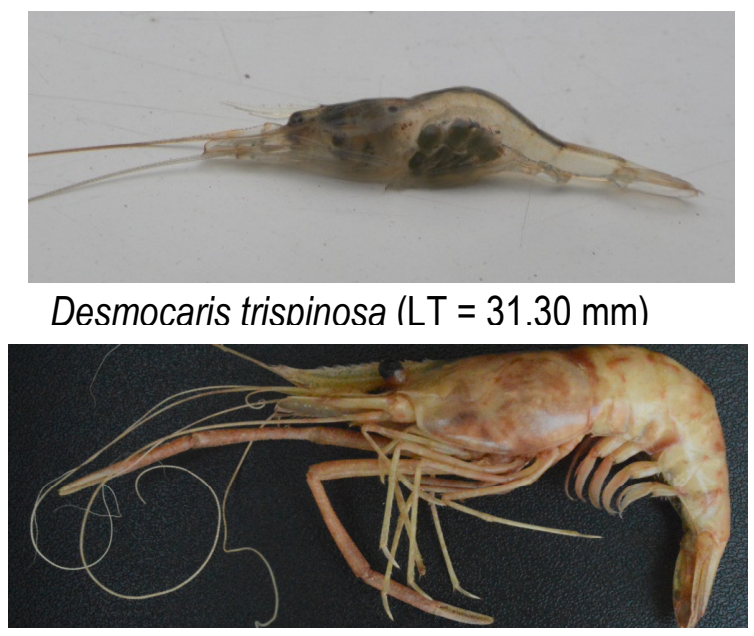

M. equidens ( $\mathrm{LT}=99,21 \mathrm{~mm}$ )

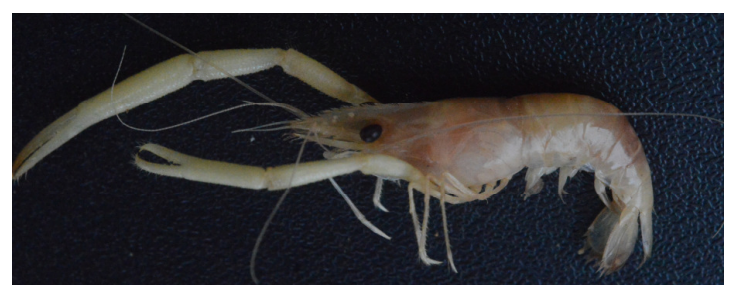

M. thysi $(\mathrm{LT}=39,13 \mathrm{~mm})$ évaluées par le test $U$ de Mann-Whitney et le test de Kruskall-Wallis a été utilisé pour comparer l'abondance des différentes espèces entre les différentes stations. L'ANOVA a permis d'évaluer le degré de significativité des variations spatiotemporelles des catégories écologiques. Le test de corrélation de Sperman a été utilisé pour l'analyse de la relation entre l'abondance des espèces et les variables environnementales. L'analyse de redondance (RDA) avec le programme Canoco for Windows version 4.1 a été employée pour mettre en relation les variables environnementales et taxons de crevettes.

trispinosa (Desmocarididae), Macrobrachium dux, $M$. equidens, M. macrobrachion, $M$. vollenhovenii et $M$. thysi (Palaemonidae) (Figure 2).

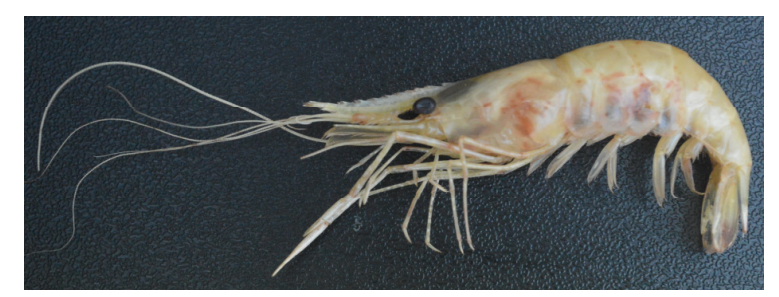

Macrobrachium dux (LT= $58.21 \mathrm{~mm})$

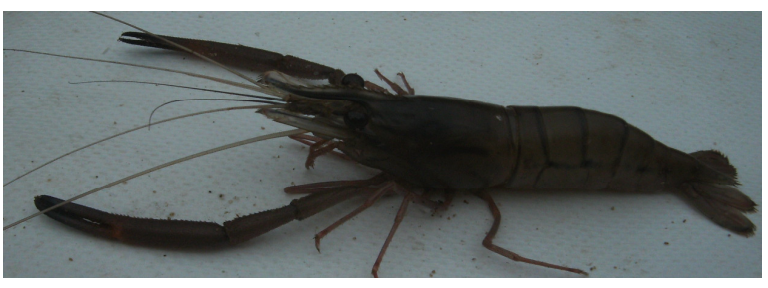

M. macrobrachion $(\mathrm{LT}=142 \mathrm{~mm})$

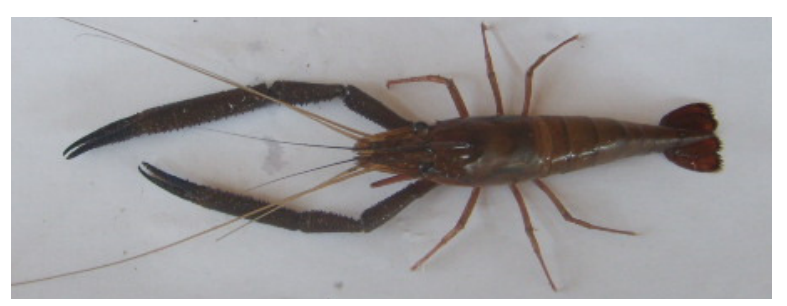

M. vollenhovenii $(\mathrm{LT}=133,56 \mathrm{~mm})$

Figure 2: Espèces de crevettes capturées dans la Forêt des Marais Tanoé-Ehy (Côte d'Ivoire) d'avril 2012 à mars 2013.

La diversité de ces espèces a varié d'une station à une autre. Elle a été plus élevée dans la station F1 avec cinq espèces. Deux espèces (Desmocaris trispinosa et $M$. thysi) ont été échantillonnées dans les stations $\mathrm{F} 3$ et $\mathrm{F} 4$, alors que $D$. trispinosa uniquement a été échantillonnée dans la station F2.
Desmocaris trispinosa a été commune aux quatre stations, $M$. thysi a été spécifique aux stations $\mathrm{F} 3$ et F4 et quatre espèces (Macrobrachium dux, $M$. equidens, M. macrobrachion, M. vollenhovenii) ont été spécifiques à la station F1. Le pourcentage d'occurrence des espèces a différé en fonction des 
stations échantillonnées. Desmocaris trispinosa a été constante dans toutes les stations (\%OF $=100)$. Macrobrachium thysi a été constante dans la station $\mathrm{F} 3(\% \mathrm{OF}=100)$ mais rare dans la station $\mathrm{F} 4(\% \mathrm{OF}=$ 8,33). Macrobrachium dux, M. equidens, $M$. macrobrachion, M. vollenhovenii ont apparu de façon accessoire $(\% \mathrm{OF}=25)$ dans la station F1. Au niveau de toutes les échantillons collectés dans cette étude dans l'ensemble des quatre stations, $D$. trispinosa a été également constante ( $\% \mathrm{OF}=100)$ pendant que $M$. thysi a été accessoire $(\% \mathrm{OF}=27,08)$. Macrobrachium dux, M. equidens, M. macrobrachion et $M$. vollenhovenii ont été des espèces rares avec une occurrence respective de 6,25\%. L'analyse de la variation saisonnière des espèces capturées a permis de montrer que Desmocaris trispinosa a apparu constante pendant les saisons sèche et pluvieuse $(\% \mathrm{OF}=100$ pour chacune des saisons). Macrobrachium dux, M. equidens, M. macrobrachion et $M$. vollenhovenii ont été accessoires pendant la saison pluvieuse $(\% \mathrm{OF}=25)$ et rares pendant la saison sèche $(\% \mathrm{OF}=0)$ dans la station $\mathrm{F} 1$. Quant à $M$. thysi, elle a été constante pendant les deux saisons dans la station F3 pendant qu'elle a demeuré rare durant la saison sèche dans la station $\mathrm{F} 4(\% \mathrm{OF}=20)$.

Abondance des espèces: Dans l'ensemble des stations échantillonnées dans la FMTE pendant la cette étude, $D$. trispinosa a été l'espèce la plus abondante avec 4823 individus sur 5227 soit 92,27 $\%$. Elle a été suivie par Macrobrachium thysi avec 329 individus soit 6,29\%, M. vollenhovenii et $M$. macrobrachion avec chacune $0,06 \%$. Les autres espèces ( $M$. dux et $M$. equidens) ont représenté 0,01 $\%$ de l'abondance totale des espèces (Figure 3). L'analyse en fonction des stations a montré que les espèces $M$. dux, M. equidens, $M$. macrobrachion et $M$. vollenhovenii ont été plus abondantes dans la station $\mathrm{F} 1, M$. thysi a été plus abondante à $\mathrm{F} 3$ et $D$. trispinosa a été abondante à F4. Le test de KruskallWallis utilisé pour comparer l'abondance des différentes espèces entre les différentes stations a montré une différence statistiquement significative pour $D$. trispinosa et $M$. thysi. L'abondance de $D$. trispinosa a différé entre la station F4 et les stations $F 2$ et $F 3$ alors que celle de $M$. thysi a différé entre la station F3 et les autres stations. L'analyse de l'abondance des différentes espèces de crevettes faite en considérant uniquement les saisons (crue ou étiage) a révélé que les espèces de grandes tailles (M. dux, M. equidens, M. macrobrachion et $M$. vollenhovenii) ont été plus abondantes pendant la période de crue alors que les espèces de petite taille (D. trispinosa et $M$. thysi) ont été plus abondantes pendant la période d'étiage (Figure 4). Seule $D$. trispinosa a présenté une abondance inter saisonnière statiquement différente (test $U$ de MannWhitney, $p<0,05)$.

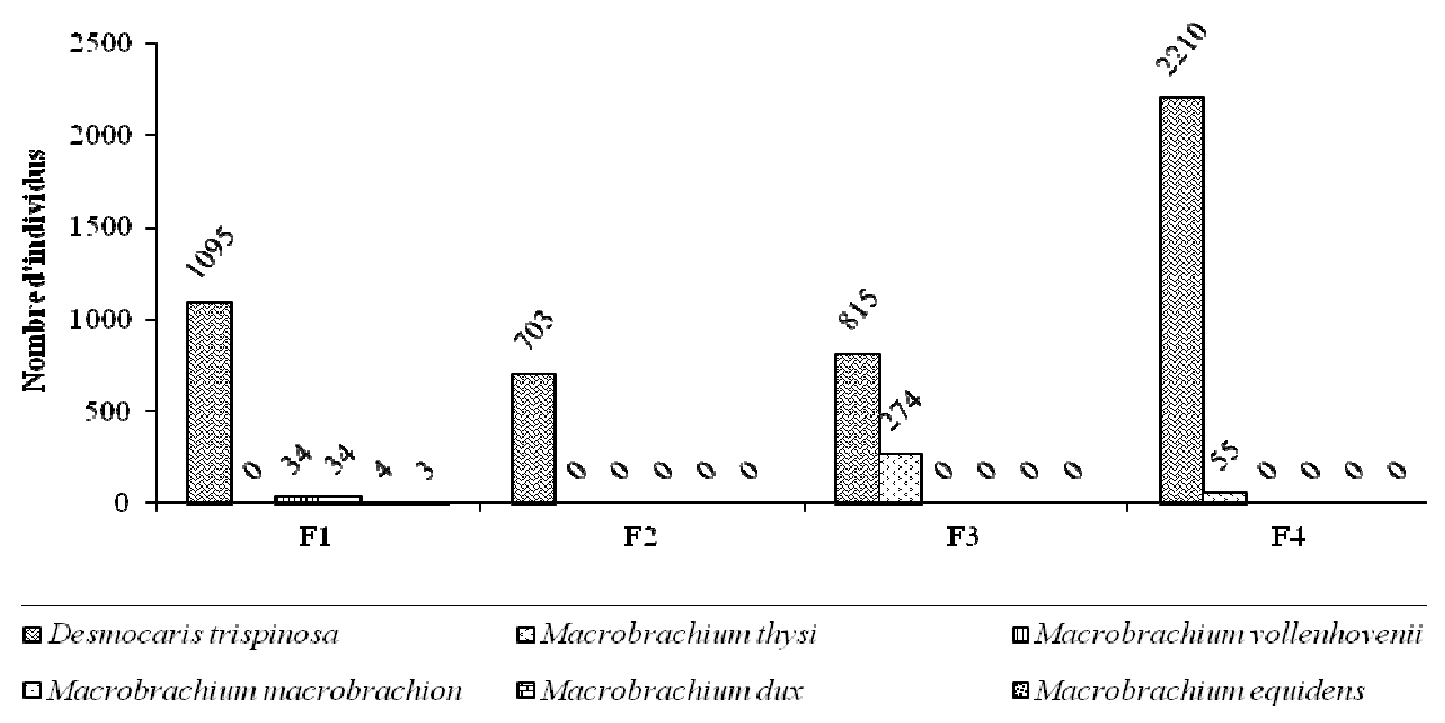

Figure 3 : Abondance des espèces de crevettes dans les différentes stations d'échantillonnage de la Forêt des Marais Tanoé-Ehy (Côte d'Ivoire) d'avril 2012 à mars 2013. 


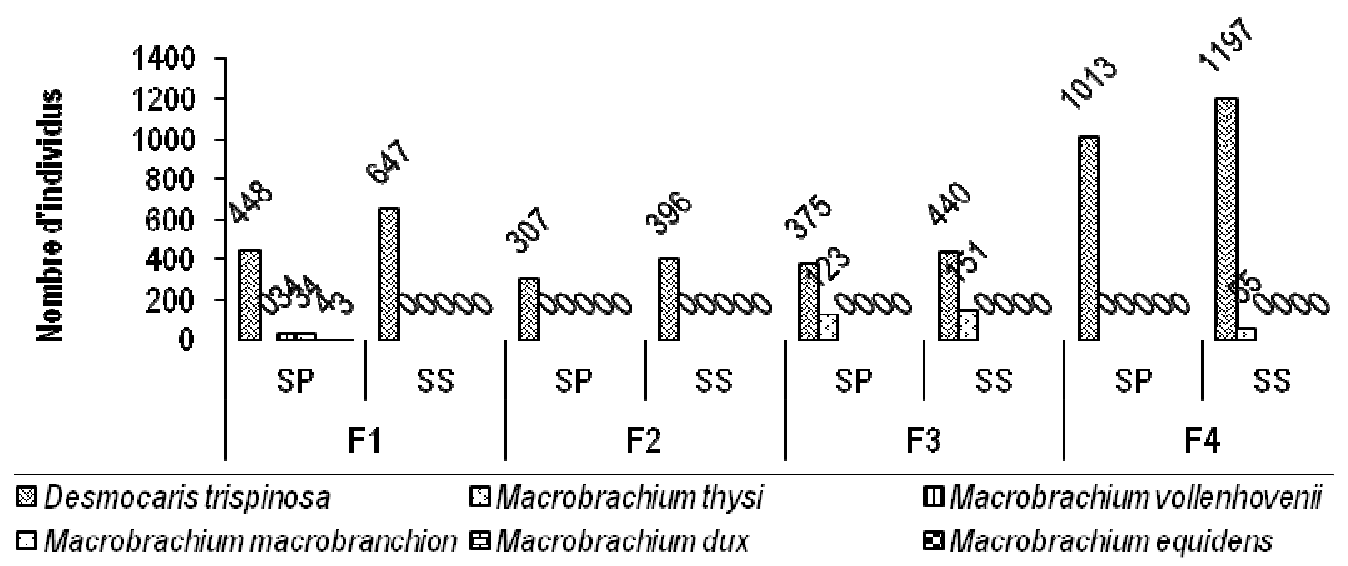

Figure 4 : Nombre des individus de crevettes capturées durant les saisons pluvieuse et sèche dans les différentes stations de la Forêt des Marais Tanoé-Ehy (Côte d'Ivoire) d'avril 2012 à mars 2013. SP = saison pluvieuse ; SS = saison sèche.

\section{Corrélations entre l'abondance des crevettes et} les variables environnementales : Les résultats de l'analyse de la corrélation de Spearman exécutée sur la matrice espèces/variables environnementales a montré que le $\mathrm{pH}$, le taux d'oxygène dissous, la conductivité, la température de l'eau, la transparence, le pourcentage du mélange sablegravier, les plantes aquatiques et le taux de fermeture de la canopée influencent fortement l'abondance des espèces de crevette $(p<0,05)$ (Tableau 2). L'abondance de $D$. trispinosa est corrélée négativement au $\mathrm{pH}$ et positivement au taux de fermeture de la canopée alors que celle de $M$. thysi est corrélée positivement à l'oxygène dissous, la transparence, le mélange sable-gravier et les plantes aquatiques. Quant aux espèces $M$. dux et $M$. vollenhovenii, elles sont négativement corrélées à la conductivité et la transparence, et positivement corrélées à la température. Les résultats de l'analyse de la redondance (RDA) exécutée sur la même matrice ont montré que les valeurs propres des axes $1(\lambda 1=0,87)$ et $2(\lambda 2=0,12)$ sont plus élevées que celles des axes $3(\lambda 3=0,01)$ et $4(\lambda 4=0,00)$. De plus, les deux premiers axes expriment la majorité de la variable cumulée espèces/variables environnementales avec 98,60\%. Par conséquent, seuls ces deux axes ont été considérés dans l'interprétation des résultats. En considérant l'axe 1 qui exprime le plus grand pourcentage de la variance cumulée $(85,6 \%)$, il y a une nette séparation de l'espèce $M$. thysi des autres espèces. Macrobrachium thysi est associée aux stations F3 et F4. Situées dans la partie négative de l'axe 1, ces stations sont caractérisées par les eaux profondes, bien oxygénées et claires avec un taux élevé du mélange sable-gravier et la présence de plantes aquatiques. Les autres espèces $[M$. dux, $M$. equidens, $M$. macrobrachion, $M$. vollenhovenii (associées à la station $\mathrm{F} 1$ ) et $D$. trispinosa (associée aux stations F1, F2 et F4)] sont corrélées positivement à la température de l'eau, au pourcentage de la boue et taux de fermeture de la canopée (Figure 5).

Tableau 2 : Résultats de l'analyse de corrélation de Spearman ( $p$-values) entre les espèces de crevettes et les variables environnementales dans la Forêt des Marais Tanoé-Ehy (Côte d'Ivoire) d'avril 2012 à mars 2013.

\begin{tabular}{lccccccccccc}
\hline & \multicolumn{10}{c}{ Variables environnementales } \\
\cline { 2 - 12 } Espèces & $\mathrm{pH}$ & $\mathrm{O}_{2}$ & $\mathrm{Cnd}$ & Prof & Te & Trans & MSG & $\mathrm{B}$ & $\mathrm{DV}$ & $\mathrm{PA}$ & $\mathrm{FC}$ \\
\hline Desmocaris trispinosa & $-\mathbf{0 , 9 1}$ & $-0,12$ & $-0,26$ & $-0,24$ & 0,33 & 0,05 & 0,08 & $-0,68$ & 0,68 & 0,08 & $\mathbf{0 , 7 7}$ \\
Macrobrachium thysi & 0,05 & $\mathbf{0 , 7 6}$ & $-0,19$ & 0,6 & $-0,68$ & $\mathbf{0 , 8 5}$ & $\mathbf{0 , 8 8}$ & $-0,50$ & 0,50 & $\mathbf{0 , 8 8}$ & $-0,62$ \\
Macrobrachium vollenhovenii & $-0,51$ & $-0,59$ & $-\mathbf{0 , 7 3}$ & $-0,06$ & $\mathbf{0 , 7 3}$ & $-\mathbf{0 , 6 6}$ & $-0,54$ & 0,25 & $-0,25$ & $-0,54$ & 0,54 \\
Macrobrachium macrobrachion & $-0,41$ & $-0,25$ & $-0,41$ & 0,25 & 0,41 & $-0,58$ & $-0,36$ & 0,17 & $-0,17$ & $-0,36$ & 0,36 \\
Macrobrachium dux & $-0,51$ & $-0,59$ & $-\mathbf{0 , 7 3}$ & $-0,06$ & $\mathbf{0 , 7 3}$ & $-0,66$ & $-0,54$ & 0,25 & $-0,25$ & $-0,54$ & 0,54 \\
Macrobrachium equidens & $-0,41$ & $-0,25$ & $-0,41$ & 0,25 & 0,41 & $-0,58$ & $-0,36$ & 0,17 & $-0,17$ & $-0,36$ & 0,36 \\
\hline
\end{tabular}

Les valeurs écrites en gras présentent une corrélation significative. 


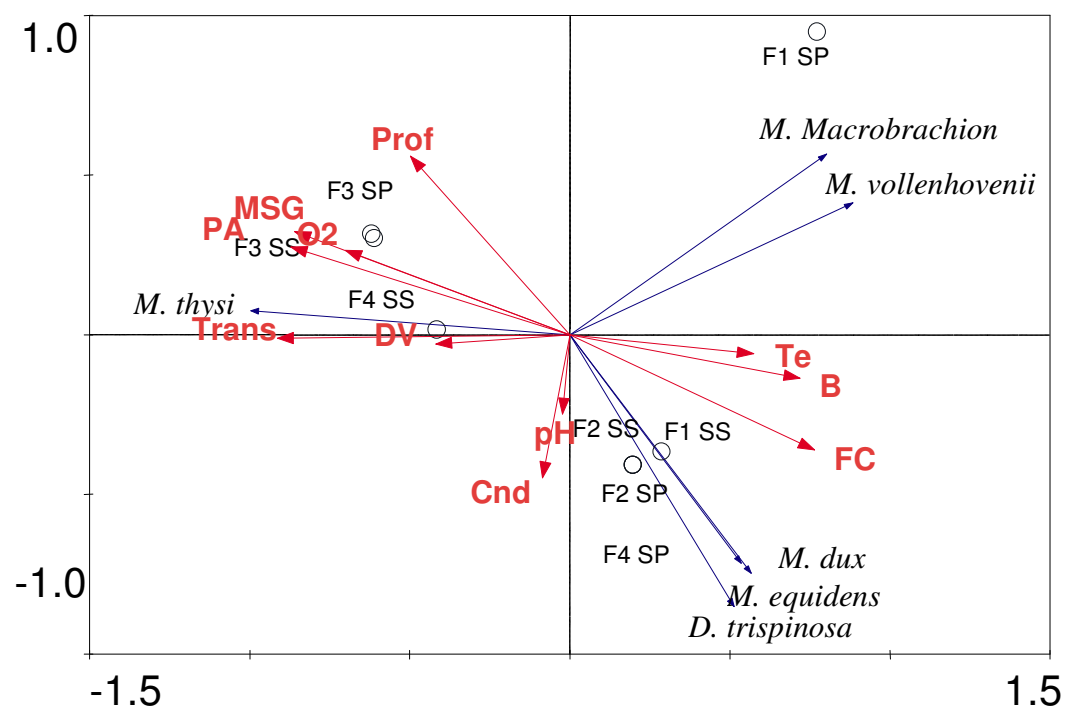

Figure 5: Résultats de l'Analyse de Redondance (RDA) montrant les relations entre les variables environnementales, les stations d'échantillonnage et les taxons de crevettes dans la Forêt des Marais TanoéEhy (Côte d'Ivoire) d'avril 2012 à mars 2013.

\section{DISCUSSION}

Les différents échantillonnages effectués dans la Forêt des Marais Tanoé-Ehy (Côte d'Ivoire) ont permis de récolter 5227 spécimens de crevettes regroupées en 6 espèces. Ces taxons appartiennent à deux familles d'eaux douces (Desmocarididae et Palaemonidae). Ces familles sont représentées chacune par un genre (Desmocaris et Macrobrachium respectivement). Le genre Macrobrachium est représenté par cinq espèces tandis que le genre Desmocaris est mono spécifique. Nos résultats sont comparables à ceux de Corredor (1979), Gooré Bi (1998) qui ont respectivement mis en évidence 6 et 7 espèces. Cependant, la richesse spécifique de la présente étude est nettement inférieure à celles mentionnées par N'Zi et al. (2007) (13 espèces) et Konan (2009) (9 espèces) mais supérieure à celles des travaux de Camara et al. (2009) et Djiriéoulou et al. (2014) qui ont fait état de 3 espèces. Plusieurs raisons pourraient expliquer des différences de compositions spécifiques: le matériel et la méthodologie utilisés, les caractéristiques environnementales des biotopes échantillonnés, les périodes d'échantillonnages et la migration des espèces. Selon Kouamélan et al. (2003), la variabilité des habitats prospectés et les périodes d'échantillonnage pourraient également justifier les différences spécifiques observées entre ces différents écosystèmes. Les grandes zones d'étude offrent une diversité d'habitats à exploiter (Graça et al., 2004). Plusieurs niches écologiques renfermant différentes espèces sont donc susceptibles d'être échantillonnées. Peut-on ajouter le matériel utilisé pour l'échantillonnage et l'effort de pêche qui sont déterminants dans la récolte. La présence de l'espèce Macrobrachium thysi dans la présente étude nuance la conclusion de Powel (1980) et Camara et al. (2009) selon laquelle cette espèce serait endémique à la forêt du Banco. En conséquence, cette espèce a une aire de distribution plus large que celle indiquée par ces auteurs. Parmi les travaux antérieurement effectués sur les crevettes des eaux douces de Côte d'lvoire, seuls ceux de N'Zi et al. (2007), Camara et al. (2009) et Djiriéoulou et al. (2014) ont signalé le genre Desmocaris dans les hydrosystèmes ivoiriens comme c'est le cas dans la présente étude. Le présent travail a enregistré la présence, uniquement pendant la crue des eaux, de quatre espèces (Macrobrachium dux, M. equidens, $M$. macrobrachion, M. vollenhovenii) dont des femelles grainées avec une espèce (Macrobrachium equidens) s'ajoutant à la liste de la faune de crevette de Côte d'lvoire. Ces espèces peuvent être qualifiées de visiteurs saisonniers ou temporaires. La présence périodique de ces espèces traduit le fait que, de part sa structuration (forte canopée) et la variabilité des biotopes, la Forêt des Marais TanoéEhy offre, non seulement une zone d'alimentation pour ces crevettes, mais aussi une zone de reproduction pour certaines espèces à cycle 
biologique complexe. L'analyse de la variation saisonnière de la richesse spécifique a montré que la plus grande richesse spécifique a été enregistrée pendant la période de crue. Ces données démontrent que les conditions de vie sont plus favorables pour les organismes aquatiques pendant cette période. En effet, en plus d'une meilleure oxygénation des eaux du fait des mouvements d'agitation dus au courant d'eau, les plans d'eau disposent aussi en période de crue, d'une importante quantité de nourriture grâce au flux de nourritures (fruits, débris végétaux et invertébrés terrestres) drainées au cours des inondations et/ou régimes des pluies (Castillo-Rivera, 2013). L'analyse faite en combinant le pourcentage d'occurrence et l'abondance des espèces a montré que, des six espèces échantillonnées dans cet écosystème, l'espèce Desmocaris trispinosa est la plus distribuée $(\% \mathrm{OF}=100)$ et la plus abondante $(92,27 \%$ d'abondance relative). En considérant les différentes saisons (sèche ou pluvieuse), elle demeure également constante et plus abondante. Elle est suivie de Macrobrachium thysi avec $\% \mathrm{OF}=27,08$ et une abondance relative de $6,29 \%$. Les espèces Macrobrachium dux, M. equidens, M. macrobrachion et $M$. vollenhovenii sont rares et moins abondantes ( $\% \mathrm{OF}=6,25$ et une abondance relative inferieure à 1

\section{CONCLUSION}

Au total six espèces de crevettes ont été échantillonnées au de cette étude. Desmocaris trispinosa apparait comme l'espèce la plus largement distribuée et la plus abondante. Le taux d'oxygène dissous, la conductivité, la température de l'eau, la transparence, le pourcentage du mélange sablegravier, les plantes aquatiques et le taux de fermeture de la canopée influencent significativement

\section{REMERCIEMENTS}

Ce travail a été réalisé dans le cadre d'un projet de recherche intitulé « Évaluation des rôles de refuges et de frayères de la Forêt des Marais Tanoé-Ehy pour les poissons des écosystèmes lagunaires et marins adjacents ". Ce dernier a bénéficié des financements du Programme d'Appui Stratégique à la Recherche Scientifique (Côte d'Ivoire) à travers

\section{EFERENCES BIBLIOGRAPHIQUES}

Adou Yao C, 2007. Inventaire préliminaire de la flore et description de la végétation de la Forêt des Marais Tanoé-Ehy. Rapport RASAP-Cl. Abidjan, Côte d'Ivoire, 29 p.
$\%$ ). La constance de $D$. trispinosa et $M$. thysi a également été observée par Camara et al. (2009). En effet, ces auteurs ont révélé que $D$. trispinosa a plus de $50 \%$ d'occurrence, une abondance relative de $67 \%$ et $M$. thysi a une occurrence et une abondance relative respective de $47 \%$ et $32 \%$ dans la rivière Banco (Côte d'lvoire). L'analyse de la corrélation entre diversité des crevettes et les caractéristiques environnementales a montré que le $\mathrm{pH}$, le taux d'oxygène dissous, la conductivité, la température de l'eau, la transparence, le pourcentage du mélange sable-gravier, les plantes aquatiques et le taux de fermeture de la canopée sont les caractéristiques environnementales qui influencent la diversité et l'abondance des espèces. Macrobrachium thysi préfère les eaux bien oxygénées, froides, à faible courant et à faible conductivité. Les mêmes tendances ont été observées pour cette espèce dans la rivière Banco (Camara et al., 2009) et dans quatre petites rivières au Sud-Est de la Côte d'Ivoire (Djiriéoulou et al., 2014). La positive corrélation entre $M$. thysi et le taux d'oxygène rend cette espèce plus sensible aux troubles de l'environnement confirmant ainsi la sensibilité de cette espèce à la dégradation de l'environnement.

la diversité et l'abondance des différentes espèces de crevettes. Compte tenu du fait que la Forêt des Marais Tanoé-Ehy abrite une espèce endémique à la Côte d'Ivoire (Macrobrachium thysi) et constitue une zone de reproduction pour plusieurs espèces dont une espèce signalée pour la première fois dans les eaux ivoiriennes ( $M$. equidens), sa préservation est vivement recommandée.

son programme " Recherche et Actions pour la Sauvegarde des Primates en Côte d'lvoire (RASAPCI). Les auteurs remercient les promoteurs du projet ainsi que les Drs Konan Yao Aristide et Koffi Barthélemy sans oublier les doctorants Simmou Yomi Junior et Money Attoubé Ida pour leur assistance.

Ahon D, 2010. Inventaire de la faune aviaire de la FMTE. Rapport RASAP-Cl et SOS Forêts, Abidjan, Côte d'Ivoire, 31 p. 
Béné JC \& Apkatou B, 2007. Inventaire préliminaire de la faune simienne de la FMTE. Rapport RASAP-Cl, Abidjan, Côte d'Ivoire, 34 p.

Camara IA, Konan MK, Diomandé D, Edia EO \& Gourène G, 2009. Ecology and diversity of freshwater shrimps in Banco National Park, Côte d'Ivoire (Banco River Basin). Knowledge and Management of Aquatic Ecosystems, 393: 1-10.

Cartes JE, Fanelli E, Papiol V \& Maynou F, 2010. Trophic relationships at intrannual spatial and temporal scales of macro and megafauna around a submarine canyon off the Catalonian coast (western Mediterranean). Journal of Sea Research, 63: 180-190.

Castillo-Rivera M, 2013. Influence of rainfall pattern in the seasonal variation of fish abundance in a tropical estuary with restricted marine communication. Journal of Water Resource and Protection, 5: 311-319.

Corredor L, 1979. Identification, distribution et aperçus écologiques des crevettes d'eau douce de Côte d'lvoire. DEA Océanographie Biologique, Université Paris 6, France, $37 \mathrm{p}$.

Dajoz R, 2000. Précis d'écologie. Dunod, Paris, France, $615 p$.

De Grave S \& Fransen CHJM, 2011. Carideorum catalogus: the recent species of the dendrobranchiate, stenopodidean, procarididean and caridean shrimps (Crustacea: Decapoda). Zoologische Mededelingen Leiden, 85 (9): 195-589.

Djiriéoulou KC, Konan KM, Koné T, Bamba $M$, Gooré Bi G \& Koné I, 2014. Shrimp Assemblages in Relation to Environmental Characteristics of Four Shallow Rivers in South East Côte d'Ivoire. Turkish Journal of Fisheries and Aquatic Sciences, 14: 651658.

Fischer W, Bianchi G \& Scott WB, 1981. Fiches FAO d'identification des espèces pour les besoins de la pêche. Atlantique centre-est. Zone de pêche 34, 47. Fonds des Nations Unies pour l'Alimentation et l'Agriculture. Ottawa, Canada, $73 p$.

Gonédélé Bi S., Koné I, Béné J-CK, Bitty AE, Akpatou BK, Gone Bi Z, Ouattara K \& Koffi DA, 2008.Tanoé forest, south-eastern Côte d'Ivoire identified as a highpriority site for the conservation of critically endangered primates in West Africa. Tropical Conservation Science, 1 (3): 265-278.

Gooré Bi G, 1998. Contribution à l'étude des crevettes d'eau douce de Côte d'Ivoire: systématique, biologie et analyse socioéconomique de la pêche de Macrobrachium vollenhovenii (Herklots 1857) et de $M$. macrobrachion (Herklots 1851) (Crustacea Decapoda, Palaemonidae) du bassin de la Bia. Thèse de Doctorat 3 e cycle, Université de Cocody-Abidjan, Côte d'Ivoire, $145 \mathrm{p}$.

Gooré $\mathrm{Bi}$ G, Kouassi NJ \&Thys Van Den Audenaerde FED, 2002. Critères pratiques d'identification et peuplement des crevettes (Caridae) de la rivière Bia (Côte d'Ivoire). Bulletin de l'Institut fondamental d'Afrique noire Cheikh Anta Diop, Dakar, Sénégal, pp. 163-186.

Gordon ND, McMahon TA \& Finlayson BL, 1994. Stream hydrology: an introduction for ecologists, Wiley and Sons, New York, France, $526 \mathrm{p}$.

Graça MAS, Pinto P, Cortes R, Coimba N, Oliveira S, Morais M, Carvalho MJ, Malo J, 2004. Factors affecting macroinvertebrate richness and diversity in Portuguese streams: a two-scale analysis. International Review of Hydrobiology, 89 (2): 151-164.

Hauhouot $C$ 2004. Les pressions anthropiques sur les milieux naturels du Sud-Est ivoirien. Revue Internationale de Géologie, de Géographie et d'Ecologie Tropicales, 28 (12): 69-82.

Konan KM, 2009. Diversité morphologique et génétique des crevettes des genres Atya Leach, 1816 et Macrobrachium Bate, 1868 de Côte d'Ivoire. Thèse de Doctorat, Université d'Abobo-Adjamé, Abidjan, Côte d'Ivoire, $154 \mathrm{p}$.

Konan YA, 2014. Diversité de l'ichtyofaune et caractéristiques bioécologiques de Clarias buettikoferi Steindachner, 1894 et Thysochromis ansorgii (Boulenger, 1901) dans la Forêt des Marais Tanoé-Ehy (Côte d'Ivoire). Thèse de Doctorat, Université Félix Houphouët-Boigny de Cocody, Abidjan, Côte d'Ivoire, $172 \mathrm{p}$.

Koné I, Béné J-CK, N'guessan KA, Bitty EA, Koffi DA, Akpatou KB \& Gonédélé Bi S, 2008. Plaidoyer pour la sauvegarde de la Forêt des Marais Tanoé-Ehy (Sud-Est de la Côte d'Ivoire), un site à valeur de conservation exceptionnelle en Afrique de l'Ouest et dans 
le monde. RASAPCI. Abidjan, Côte d'Ivoire, $25 \mathrm{p}$.

Koné T, Koné I \& Koffi AD, 2009. Fish diversity in an unique environnemental complex: Tanoé Forest-Lower stream of Tanoé River-Ehy Lagoon. Poster publicitaire.

Kouamélan EP, Teugels GG, N'Douba V, Gooré Bi $G$ \& Koné $T, 2003$. Fish diversity and its relationships with environmental variables in a West African basin. Hydrobiologia, 505:139-146.

Kpan TF, 2012. Peuplement des amphibiens anoures de la Forêt des Marais Tanoé-Ehy (Sud-Est Côte d'Ivoire). Diplôme d'Études Approfondies, Université Félix HouphouëtBoigny de Cocody, Abidjan, Côte d'Ivoire, $57 \mathrm{p}$.

Missa K, Ouattara DN, Koné M \& Bakayoko A, 2015. Étude floristique et diversité de la Forêt des Marais Tanoé-Ehy (Sud- Est de la Côte d'Ivoire). Journal of Animal \& Plant Sciences, 253: 3917-3938.Monod T, 1966. Crevettes et crabes des côtes occidentales d'Afrique. Mémoires, 77: 103-234.

Monod T, 1980. Décapodes. In: Flore et faune aquatiques de l'Afrique sahélo-soudanienne (Durand JR \& Lévêque C., eds). ORSTOM, Paris, France, Tome I, 44: 369-389.

N'Zi KG, 2007. Diversité biologique des peuplements de crevettes d'eaux douces de Côte d'Ivoire en relations avec les variables environnementales du milieu. Thèse de Doctorat, Université de Cocody-Abidjan, Côte d'Ivoire, $178 \mathrm{p}$.

Powell CB, 1980. The genus Macrobrachium in West Africa. In: M. thysi, a new largeegged species from Ivory Coast (Crustacea Decapoda Palaemonidae). Revue de Zoologie africaine, 94: 317-326.

Powell CB, 1982. Fresh and brackish water shrimps of economic important in the Niger Delta. University of Port Harcourt. Present to the second conference of the fisheries socierty of Nigeria held at Calabar, 24-27 January, 1982, 1-45.

Rafalimanana T, 2003. Les crevettes Pénéidés exploitées sur la côte ouest de Madagascar : variabilités spatio-temporelles des paramètres biologiques et dynamique des populations. Thèse de Doctorat, Université de Rennes 1, France, $268 \mathrm{p}$.

Rios SL \& Bailey RC, 2006. Relationships between riparian vegetation and stream benthic communities at three spatial scales. Hydrobiologia, 553: 153-160.

Short JW, 2004. A revision of Australian river prawns, Macrobrachium (Crustacea: Decapoda: Palaemonidae). Hydrobiologia, 525: 1-100. 